\title{
FERMT1 knockdown inhibits oral squamous cell carcinoma cell epithelial-mesenchymal transition by inactivating the PI3K/AKT signaling pathway
}

\author{
Xiao Wang ${ }^{1,2}$ and Qianqian Chen ${ }^{2^{*}}$
}

\begin{abstract}
Background: The metastasis of oral cancer is one of the main causes of death. However, the mechanisms underlying oral cancer metastasis have not been completely elucidated. Fermitin family member 1 (FERMT1) plays an -oncogene role in many cancers; however, the role of FERMT1 in oral squamous cell cancer (OSCC) remains unclear.

Methods: In this study, OSCC cells were treated with $5 \mathrm{ng} / \mathrm{ml}$ recombinant human Transforming growth factor- $\beta 1$ (TGF- $\beta 1$ ) protein. FERMT1 expression was measured in OSCC cell lines by RT-qPCR and western blotting. The effect of FERMT1 knockdown on the migration and invasion of OSCC cells was evaluated by Transwell assay. The epithelialmesenchymal transition (EMT) and PI3K/AKT signaling pathway-related mRNA expression and protein levels were assessed by RT-qPCR and western blotting.

Results: We found that FERMT1 expression was elevated in TGF- $\beta 1$-induced OSCC cell lines, and knockdown of FERMT1 inhibited the migration and invasion in TGF- $\beta 1$-induced OSCC cells. FERMT1 silencing inhibited vimentin, $\mathrm{N}$-cadherin, matrix metalloproteinase 9 (MMP-9) expression and promoted E-cadherin expression, suggesting that FERMT1 silencing inhibited EMT in TGF- $\beta 1$-induced OSCC cells. Furthermore, FERMT1 silencing inactivated the PI3K AKT signaling pathway in TGF- $\beta 1$-induced OSCC cells. Activation of the PI3K/AKT signaling pathway reversed the effect of FERMT1 silencing on OSCC cell migration, invasion, and EMT.
\end{abstract}

Conclusions: FERMT1 silencing inhibits the migration, invasion, and EMT of OSCC cells via inactivation of the PI3K/ AKT signaling pathway, suggesting that FERMT1 is a novel and potential therapeutic target for anti-metastatic strategies for OSCC.

Keywords: FERMT1, Invasion, Migration, Oral squamous cell cancer

\section{Background}

Oral cancer is the eighth most common type of cancer worldwide, and approximately $90 \%$ of all oral malignancies are oral squamous cell cancer (OSCC) [1]. In

\footnotetext{
*Correspondence: qianqianchen@gxust.edu.cn

${ }^{2}$ Medical College, Medical Experimental Center, Guangxi University of Science and Technology, Building D, 257 Liushi Road, Yufeng District, Liuzhou 545006, China

Full list of author information is available at the end of the article
}

China, the overall crude incidence rate for oral cancer was 2.93/100,000 in 2011 [2]. Smoking, alcohol drinking, human papilloma virus, serum excess and deficient levels of $\mathrm{Cu}$ or $\mathrm{Zn}$, and betel quid (BQ) chewing play key roles in the development of oral cancer [3-7]. At present, the mortality rate of oral cancer remains high, and the primary cause is tumor metastasis $[8,9]$. Hence, knowing what genes can inhibit oral cancer metastasis and EMT can help the development of therapeutic targets. 
Epithelial-mesenchymal transition (EMT) occurrence help the OSCC metastasis [10, 11]. During EMT, epithelial cells lose their junctions and increase their motility, which promotes epithelial cells to leave the tissue and enter the systemic circulation, thereby enhancing the development of cancer metastasis [12]. During EMT, there is a decrease in the expression of epithelial markers, including E-cadherin, tight junction proteins, and cytokeratin, as well as increase in the expression of mesenchymal markers, including vimentin, fibronectin, $\alpha$-smooth muscle actin, $\mathrm{N}$-cadherin [12]. EMT programs can be activate by activated PI3K/Akt pathway in oral cancer [13, 14]. PI3K/Akt pathway play a key role in regulating cell growth and metabolism in normal physiology. PI3K can activate by insulin-like growth factor 1 receptor, then induced phosphorylation of AKT [15-17]. AKT which is a central mediator of the PI3K pathway can further activate the phosphorylation of mTOR and NF- $\mathrm{kB}$ and regulate eukaryotic translation initiation factor $4 \mathrm{E}$ and ribosomal protein S6 kinase in cancer [15-17]. Hence, excessively increased PI3K expression and the level of phosphorylated AKT/ total AKT (p-AKT/AKT) indicates activation of the PI3K/AKT signaling pathway. In addition, the activation of EMT programs involves many proteins, such as fermitin family member 1 (FERMT1). FERMT1, which encodes the kindlin-1 protein that belongs to a family of focal adhesion proteins, activates EMT to promote colon cancer metastasis both in vitro and in vivo [18]. However, whether FERMT1 can promote OSCC metastasis and EMT by activating PI3K/AKT have not been explored.

In OSCC, EMT was induced by TGF- $\beta 1$ [19-21]. In this study, FERMT1 expression was measured in OSCC cell lines with or without TGF- $\beta 1$ induction. Additionally, the effect of FERMT1 knockdown on the migration, invasion, EMT, and PI3K/Akt pathway in OSCC cells were studied. Our research explored and confirmed the role of FERMT1 as a therapeutic target for OSCC metastasis.

\section{Materials and methods Cell culture}

Human oral keratinocytes (HOK) and OSCC cell lines include CAL-27, Tca8113, and SCC15 (China Center for Type Culture Collection, Wuhan, China) were cultured in the special keratinocyte growth medium (Clonetics, San Diego, CA, USA) and the Dulbecco's modified Eagle medium supplemented with $10 \%$ fetal bovine serum, respectively. All cells were treated with $5 \mathrm{ng} / \mathrm{ml} \mathrm{recom-}$ binant human TGF- $\beta 1$ protein (T \& L biological technology, Beijing, China).

\section{Reverse transcription-quantitative polymerase chain reaction (RT-qPCR)}

Total RNA was extracted from TGF- $\beta 1$-treated Tca8113 and SCC15 cells using the TRIzol ${ }^{\circledR}$ reagent (Invitrogen; Thermo Fisher Scientific, Inc., Foster City, CA, USA), reversed transcription using the PrimeScript RT Reagent kit, and FERMT1 expression was measured by RT-qPCR using SYBR Premix Ex Taq reagent (Takara, Dalian, China). RT-qPCR was performed using an Applied Biosystems 7500 system (ThermoFisher Scientific). The primer sequences are presented in Table 1. Gene expression levels were quantified using the $2^{-\Delta \Delta C t}$ method, and normalized to the expression of the control, GAPDH (13).

\section{Western blotting}

FERMT1, PI3K, AKT, p-AKT, MMP-9, E-cadherin, vimentin, and $\mathrm{N}$-cadherin expression were measured by western blotting according to previous study [21]. Briefly, the total protein was extracted from TGF- $\beta 1$-treated Tca8113 and $\mathrm{SCC} 15$ cells using radioimmunoprecipitation assay buffer (Takara), separated by $8 \%$ SDS-PAGE, and then transferred onto polyvinylidene difluoride membranes. After blocking, the membranes were incubated with rabbit

Table 1 The primer sequences

\begin{tabular}{|c|c|c|c|}
\hline Gene & Forward primer $\left(5^{\prime}-3^{\prime}\right)$ & Reverse primer $\left(5^{\prime}-3^{\prime}\right)$ & Size \\
\hline FERMT1 & TAAACTTGCAGATAATCTCA & CAAGTTCCTTATTTTTAAAG & $118 \mathrm{bp}$ \\
\hline PI3K(PIK3CB) & TAATCGGAGGATAGGGCAGT & TTCATGTGCCCCACACTTCC & $123 \mathrm{bp}$ \\
\hline AKT (AKT1) & GGTGATCCTGGTGAAGGAGA & AAGGGGTGCCTGGAGTTCTG & $138 \mathrm{bp}$ \\
\hline MMP-9 & TCTGCCTGCACCACCGACG & CTGGGTGTAGAGTCTCTCG & $114 \mathrm{bp}$ \\
\hline E-cadherin & CAACGATAATCCTCCGATCT & ACGGTGACGGTGGCTGTGGA & $138 \mathrm{bp}$ \\
\hline vimentin & GAAGAGGAAATCCAGGAGCT & TTTCATATTGCTGACGTACGT & $118 \mathrm{bp}$ \\
\hline $\mathrm{N}$-cadherin & TAATGGAAATCAAGT & ATCCCTCAGGAACTGTCCCA & $113 \mathrm{bp}$ \\
\hline GAPDH & GCTCATTTGCAGGGGGGAG & GTTGGTGGTGCAGGAGGCA & $138 \mathrm{bp}$ \\
\hline
\end{tabular}


anti-human FERMT1 (ab68041), PI3K (ab140307), AKT (ab8805), p-AKT (ab38449), MMP-9 (ab76003), E-cadherin (ab76055), vimentin (ab92547), N-cadherin (ab76011), and GAPDH (ab8245) antibody (Abcam, Cambridge, MA, USA) for $1 \mathrm{~h}$ at $37^{\circ} \mathrm{C}$. After wash, membranes were incubated with goat anti-rabbit secondary antibody (a horseradish peroxidase-conjugated IgG H\&L, Abcam) for $40 \mathrm{~min}$, and proteins were visualized using an enhanced chemiluminescence reagent (Thermo Fisher Scientific, Inc.). The expression levels of the proteins of interest were normalized (Image J 6.0, National Institutes of Health, Bethesda, MD, USA) against the expression levels of $\beta$-actin.

Transfection with si-FERMT1 and IGF-1 treatment siRNA negative control (si-NC, 5'-UUCUCCGAACGU GUCACGUTT-3') and si-FERMT1 (si-FERMT1-1: 5'-GAAACAAGUGCUAAGUGUACC-3'; si-FERMT1-2: 5'-CUAUUUCUCAGUUCUAUUAUU-3'; si-FERMT1-3: 5'-GGGAAAUAUCAGACAAUAUUU-3') were purchased from GenePharma (Suzhou, China). Cells were transfected with 20,50, and $100 \mathrm{nM}$ siRNAs using the Lipofectamine ${ }^{\circledR}$ 2000 reagent (Invitrogen; Thermo Fisher Scientific). Then the si-FERMT1-transfected cells were treated with PBS or insulin-like growth factor-1 (IGF-1).

\section{Transwell migration and invasion assays}

Cell migration and invasion were assessed using the Transwell assay with or without Matrigel pre-coating (BD Biosciences), respectively, according to previous study [21]. After culturing for $24 \mathrm{~h}$, cells from the bottom chamber were stained with $0.1 \%$ crystal violet solution in $20 \%$ ethanol, and counted using a phase contrast light microscope (Olympus Corporation, Tokyo, Japan). The migrated and invasive cells were calculated in 5 randomly selected highpower fields and the mean was as the final result.

\section{Statistical analysis}

Data in this study conformed to normal distribution are presented as mean \pm standard deviation. The differences between groups were performed using the SPSS software version 19.0 (IBM SPSS, Armonk, NY, USA). The differences between the two groups were used to analyze an independent t-test. While the differences between three or more groups were analyzed using a one-way analysis of variance, followed by a post-hoc LSD test. $P<0.05$ has a significant difference.

\section{Results}

FERMT1 expression is promoted in in TGF- $\beta 1$-induced OSCC cell lines

FERMT1 expression was measured by RT-qPCR and western blotting (Fig. 1). Compared with control group, FERMT1 mRNA expression and protein level was
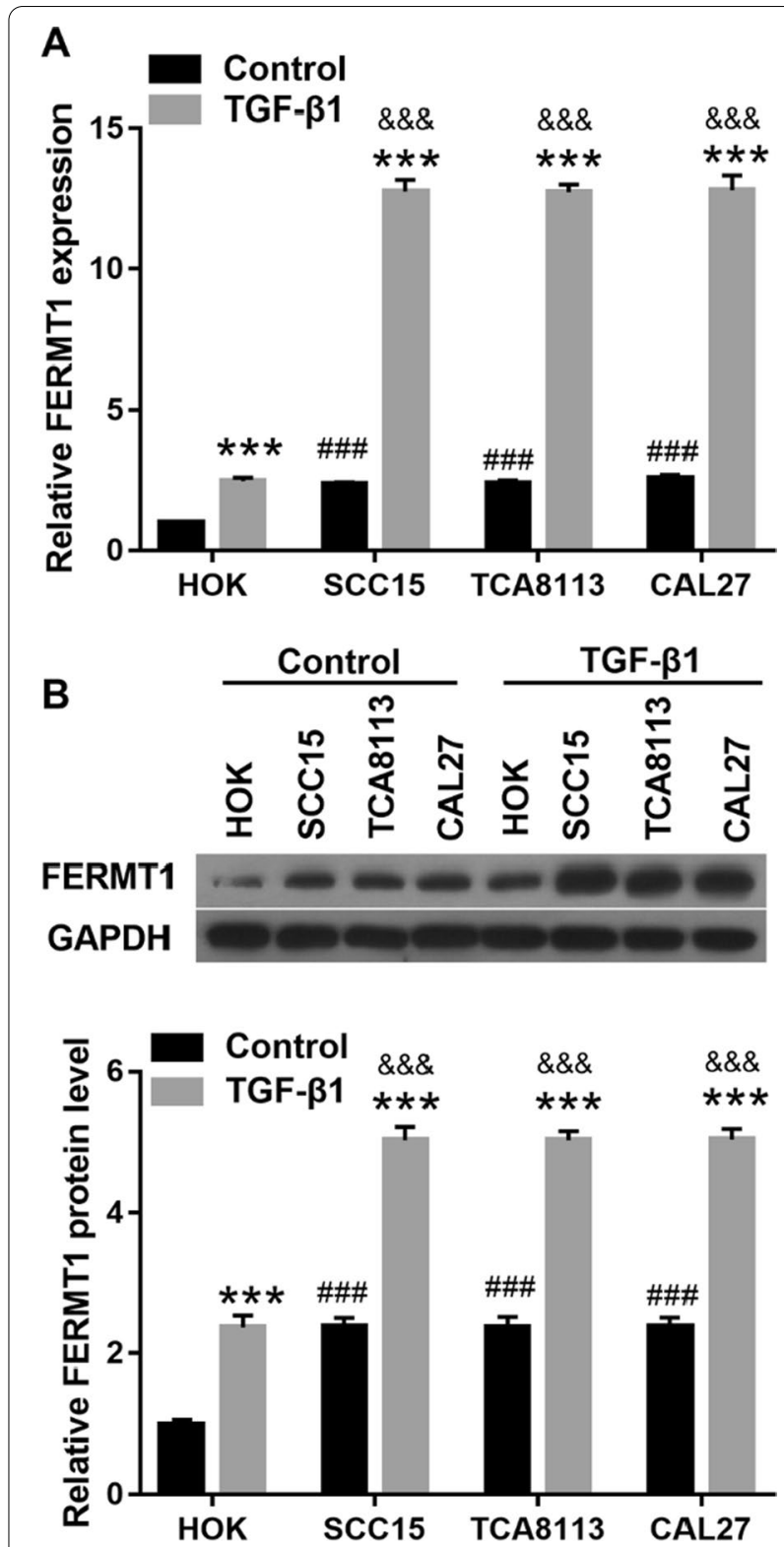

Fig. 1 FERMT1 expression is promoted in oral squamous cell cancer cell lines. OSCC cells and HOK cell were treated with $5 \mathrm{ng} /$ $\mathrm{ml}$ recombinant human TGF- $\beta 1$ protein. FERMT1 expression was analyzed by RT-qPCR (a) and western blotting (b). ${ }^{* *} P<0.001$ TGF- $\beta 1$ treatment group vs control group. ${ }^{\# \#} P<0.001$, OSCC cells vs HOK cell in control group. ${ }^{\& \&} \&<<0.001$, OSCC cells vs HOK cell in TGF- $\beta 1$ treatment group. HOK: human oral keratinocytes; CAL-27, Tca8113, and SCC15: oral squamous cell cancer cell lines

significantly enhanced in the in TGF- $\beta 1$-induced HOK, CAL-27, Tca8113, and SCC15. Compared with HOK in control group, FERMT1 expression was significantly enhanced in the in OSCC cell lines CAL-27, Tca8113, and SCC15 in control group. In addition, FERMT1 expression was significantly enhanced in the in TGF- $\beta 1$-induced 
OSCC cell lines CAL-27, Tca8113, and SCC15 compared with TGF- $\beta 1$-induced HOK. FERMT1 expression did not differ significantly between the three OSCC cell lines. Hence, Tca8113 and SCC15 cells were selected for further experiments.

\section{Knockdown of FERMT1 suppresses migration and invasion} in TGF- $\beta 1$-induced OSSC cells

To assess the effect of FERMT1 on OSCC cell migration and invasion, si-FERMT1s were transfected into Tca8113 and SCC15 cells, and FERMT1 expression was measured by RT-qPCR and western blotting (Fig. 2). Compared with other groups, FERMT1 expression was significantly inhibited in the 100-nM si-FERMT1-1 (named si-FERMT1 in further experiments) group. Following transfection of si-FERMT1 or si-NC at $48 \mathrm{~h}$, the migration and invasion of Tca8113 and SCC15 cells was significantly reduced in the si-FERMT1 group compared with the si-NC group (Fig. 3). Additionally, western blotting results showed that si-FERMT1 transfection evidently inhibited vimentin, N-cadherin, and MMP-9 mRNA expression and protein level while promoted E-cadherin mRNA expression and protein level compared with siNC-transfected Tca8113 and SCC15 cells (Fig. 4).

\section{Knockdown of FERMT1 silences the PI3K/AKT signaling pathway}

After transfection with si-FERMT1 and si-NC, the PI3K mRNA expression was markedly lower while t-AKT had no marked change in si-FERMT1-transfected Tca8113 and SCC15 cells compared with that in si-NC transfected Tca8113 and SCC15 cells. In addition, the protein levels of PI3K and p-AKT and p-AKT/t-AKT were markedly lower while t-AKT had no marked change in si-FERMT1-transfected Tca8113 and SCC15 cells compared with those in si-NC transfected Tca8113 and SCC15 cells (Fig. 4).

\section{Activated PI3K/AKT signaling pathway reverses the effect of FERMT1 on OSSC cell migration and invasion}

To determine whether FERMT1 contributed to OSSC migration and invasion via the PI3K/AKT signaling pathway, we activated the PI3K/AKT signaling pathway by treatment with IGF-1 (the PI3K/AKT signaling pathway activator) in si-FERMT1-transfected Tca8113 and SCC15 cells. After $48 \mathrm{~h}$ of treatment, the expression of PI3K and p-AKT was markedly higher in the IGF-1 treatment group than that in the PBS treatment group in siFERMT1-transfected Tca8113 and SCC15 cells (Fig. 5). The migration and invasion of si-FERMT1-transfected Tca 8113 and SCC15 cells were significantly promoted by IGF-1 treatment compared with PBS treatment (Fig. 6). Additionally, IGF-1 treatment significantly enhanced

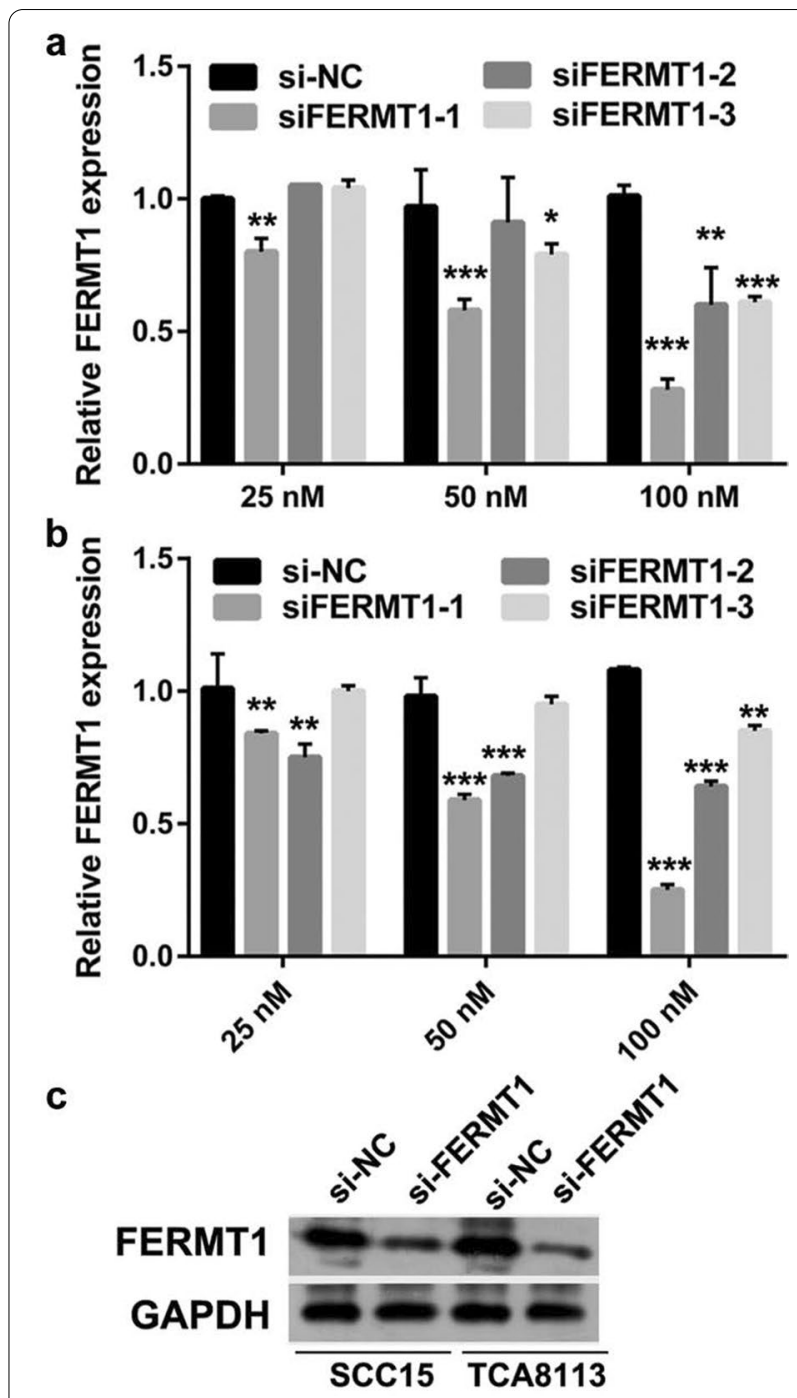

Fig. 2 FERMT1 expression was silenced by si-FERMT1 transfection. OSCC cells were treated with $5 \mathrm{ng} / \mathrm{ml}$ recombinant human TGF- $\beta 1$ protein. (a and $\mathbf{b})$ FERMT1 expression was analyzed by RT-qPCR after transfection of three si-FERMT1s at $24 \mathrm{~h}$ in SCC15 (a) and Tca8113 (b) cells. (c) FERMT1 expression was analyzed by western blotting. ${ }^{*} P<0.05,{ }^{* *} P<0.01$, and ${ }^{* *} P<0.001$ vs. si-NC

vimentin, N-cadherin, and MMP-9 mRNA expression and protein level while reduced E-cadherin mRNA expression and protein level compared with PBS treatment in si-FERMT1-transfected Tca8113 and SCC15 cells (Fig. 7).

\section{Discussion}

The metastasis of oral cancer is one of the main causes of death $[8,9]$. However, the mechanisms underlying oral cancer metastasis remain to be elucidated. In this study, we found that FERMT1 expression was elevated in TGF- $\beta 1$-induced OSCC cell lines, and knockdown of 

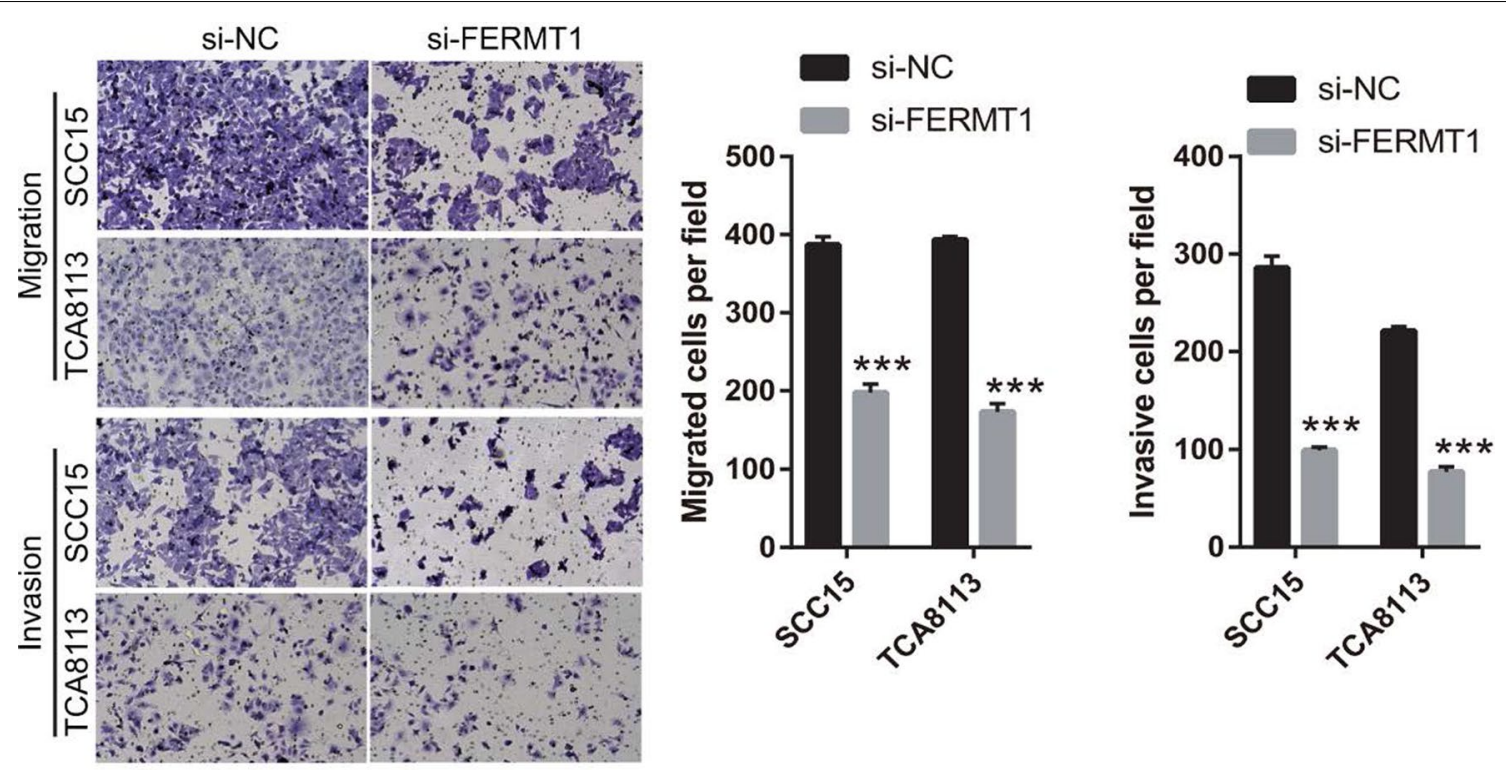

Fig. 3 Knockdown of FERMT1 suppresses migration and invasion of OSSC cells. OSCC cells were treated with 5 ng/ml recombinant human TGF- $\beta 1$ protein. The migration and invasion of OSSC cells were measured by Transwell $(\times 200)$. ${ }^{* *} P<0.001$
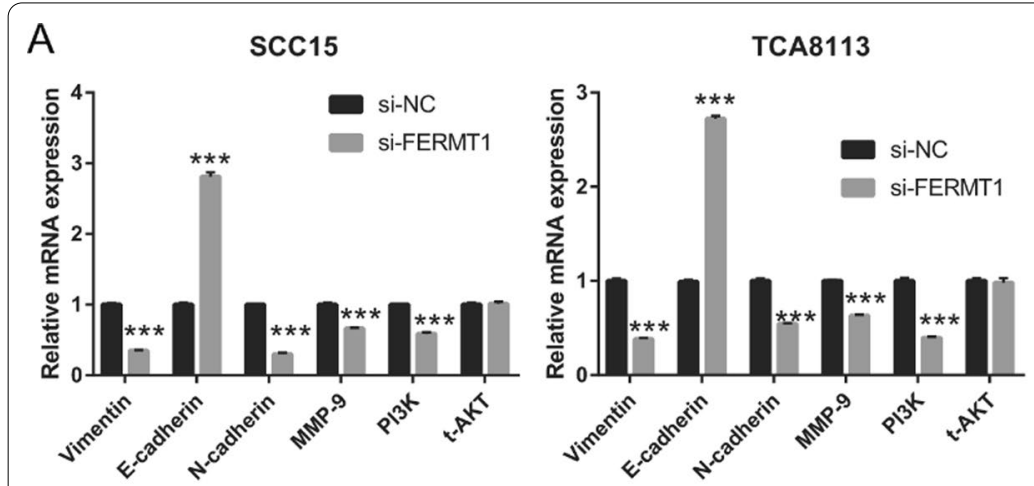

B
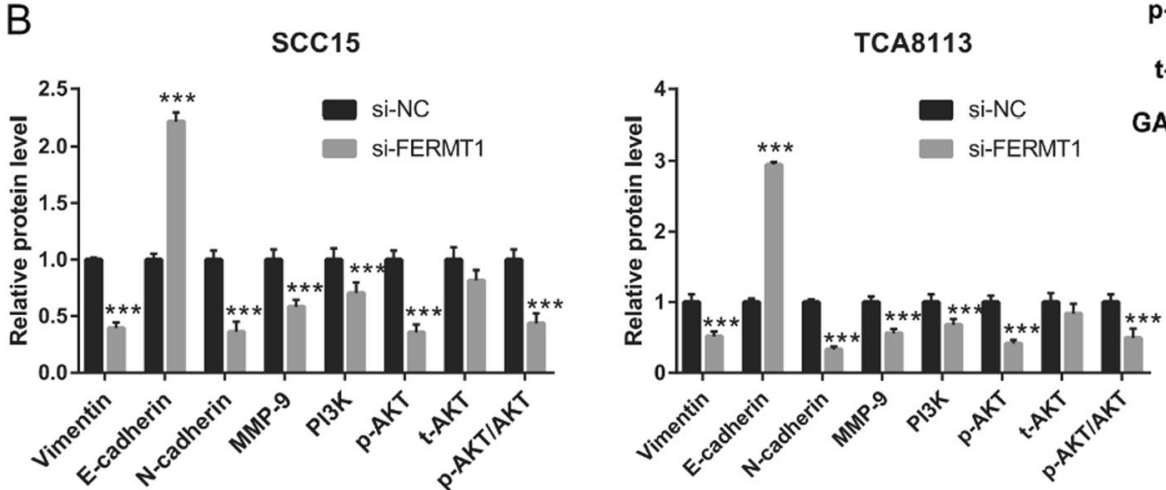

C

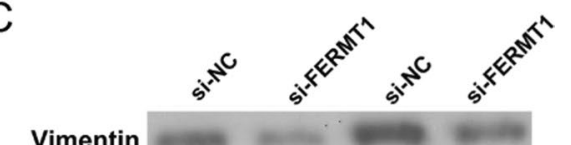

N-cadh

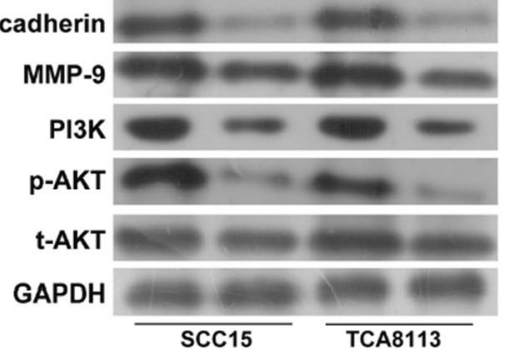

Fig. 4 Knockdown FERMT1 inhibited EMT and the PI3K/AKT signaling pathway. OSCC cells were treated with $5 \mathrm{ng} / \mathrm{ml}$ recombinant human TGF- $\beta 1$ protein. (A, B) mRNA Expression (A) and protein levels (B) of mesenchymal biomarkers including vimentin, N-cadherin, and MMP-9, epithelial biomarkers including E-cadherin, and PI3K, total (t)-AKT, and phosphorylated (p)-AKT were measured by qRT-PCR and western blotting after transfection in Tca8113 and SCC15 cells at $24 \mathrm{~h}$. (C) The representative image of western blot 

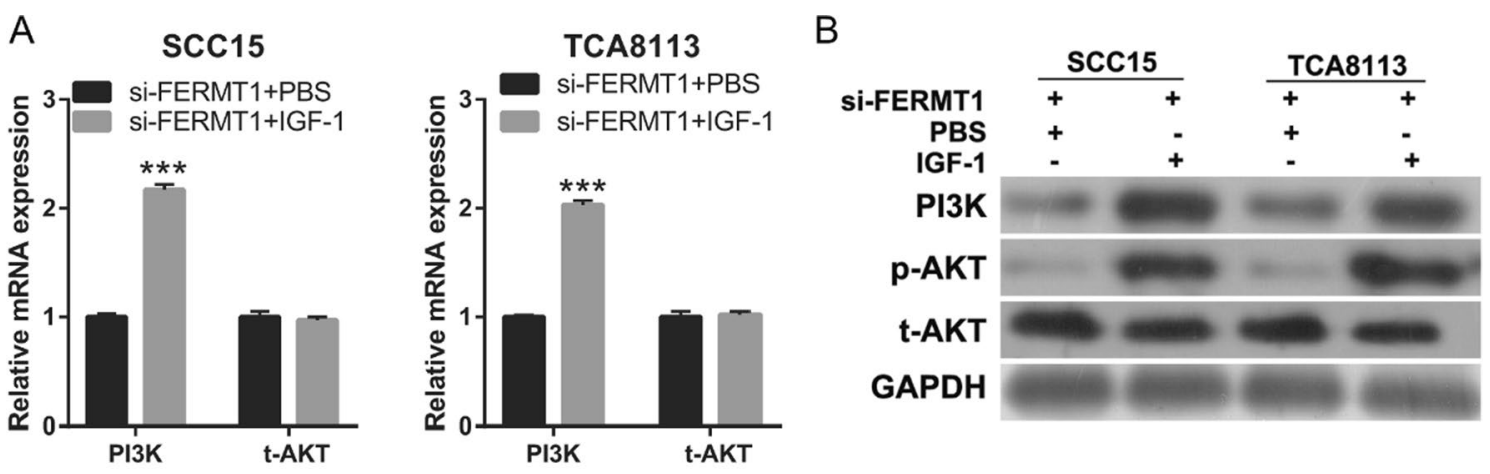

C
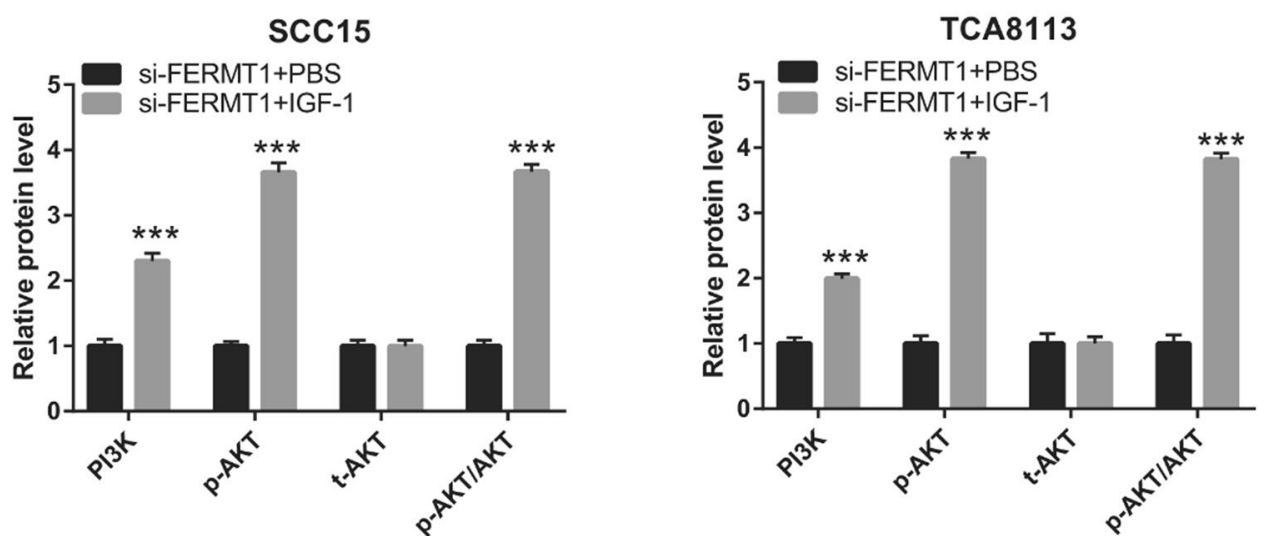

Fig. 5 IGF-1 treatment activated the PI3KJAKT signaling pathway in si-FERMT1-transfected Tca8113 and SCC15 cells. OSCC cells were treated with $5 \mathrm{ng} / \mathrm{ml}$ recombinant human TGF- $\beta 1$ protein. A PI3K, total (t)-AKT mRNA expression were analyzed by qRT-PCR after IGF-1 treatment at $24 \mathrm{~h}$ in si-FERMT1-transfected Tca8113 and SCC15 cells. B The representative image of western blot. C PI3K, t-AKT, and phosphorylated (p)-AKT protein levels were analyzed by western blotting after IGF-1 treatment at $24 \mathrm{~h}$ in si-FERMT1-transfected Tca8113 and SCC15 cells. ${ }^{* * *} P<0.001$, si-FERMT1 + IGF-1 vs si-FERMT1 + PBS

FERMT1 inhibited the migration, invasion, and EMT in TGF- $\beta 1$-induced OSCC cells. Notably, we revealed that FERMT1 activated the PI3K/AKT signaling pathway to promote EMT in OSCC metastasis.

FERMT1 encodes the kindlin-1 protein, which is a focal adhesion protein. A previous study found that FERMT1 expression was increased in colon cancer, which was an independent prognostic factor for poor overall survival, and FERMT1 promoted colon cancer metastasis [22, 23]. FERMT1 expression was correlated with metastasis-free survival in breast cancer, and silencing of FERMT1 inhibited lung metastasis of breast cancer [24]. FERMT1 expression was also increased in pancreatic cancer, and FERMT1 promoted pancreatic cancer metastasis [25]. These results suggest that FERMT1 expression is elevated in cancer, and that knockdown of FERMT1 expression can inhibit the migration and invasion of cancer cells. Similar to the findings of these results, we found that FERMT1 expression was elevated in TGF- $\beta 1$-induced OSCC cell lines, and knockdown of FERMT1 inhibited the migration and invasion in the TGF- $\beta 1$-induced OSCC cells.

EMT plays an important role in initiating metastasis. When EMT occurs, expression levels of mesenchymal biomarkers, including $\mathrm{N}$-cadherin, vimentin, and MMP-9 are increased, and those of epithelial biomarkers such as E-cadherin are reduced [26]. FERMT1 mediates the $\beta$-catenin/EMT signaling pathway to promote colon cancer metastasis [27]. Additionally, Kindlin-1 is required for colorectal cancer cell migration and invasion via activation of the TGF- $\beta / \mathrm{Smad} 3$ signaling pathway and EMT [28]. Higher FERMT1 expression was found in human gastric cancer tissues and has been significantly associated with poor overall survival; FERMT1 enhanced gastric cancer metastasis and EMT by activating the NF-kB pathway [29]. These studies suggested that FERMT1 promoted cancer progression by enhancing many signaling pathways and EMT. Similar to the findings of these studies, we found that silencing of FERMT1 markedly inhibited vimentin, N-cadherin, and MMP-9 expression and promoted 

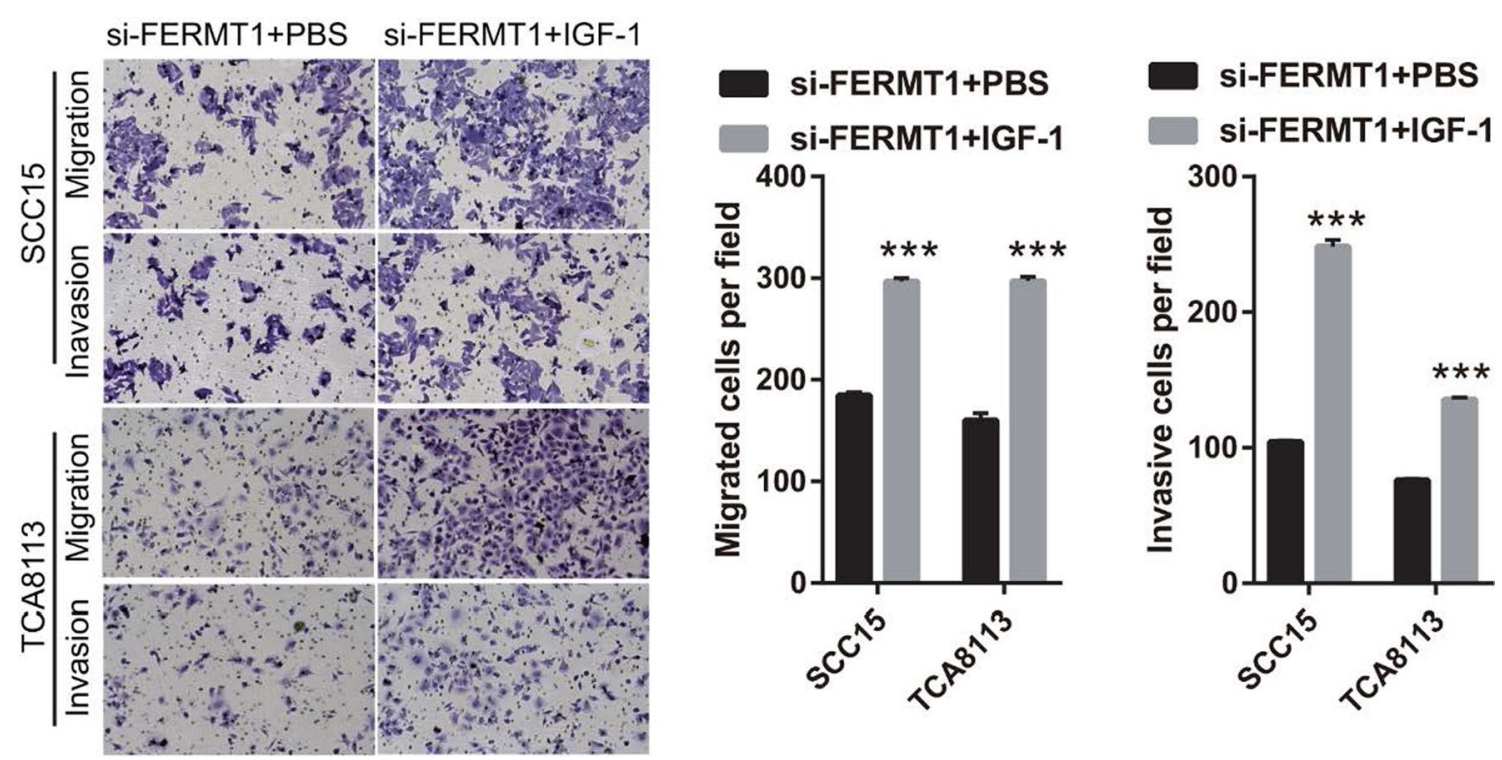

Fig. 6 Migration and invasion were promoted after IGF-1 treatment at $24 \mathrm{~h}$ in si-FERMT1-transfected Tca8113 and SCC15 cells. OSCC cells were treated with $5 \mathrm{ng} / \mathrm{ml}$ recombinant human TGF- $\beta 1$ protein. The migration and invasion of OSSC cells were measured by Transwell ( $\times 200)$. ${ }^{* * *} P<0.001$

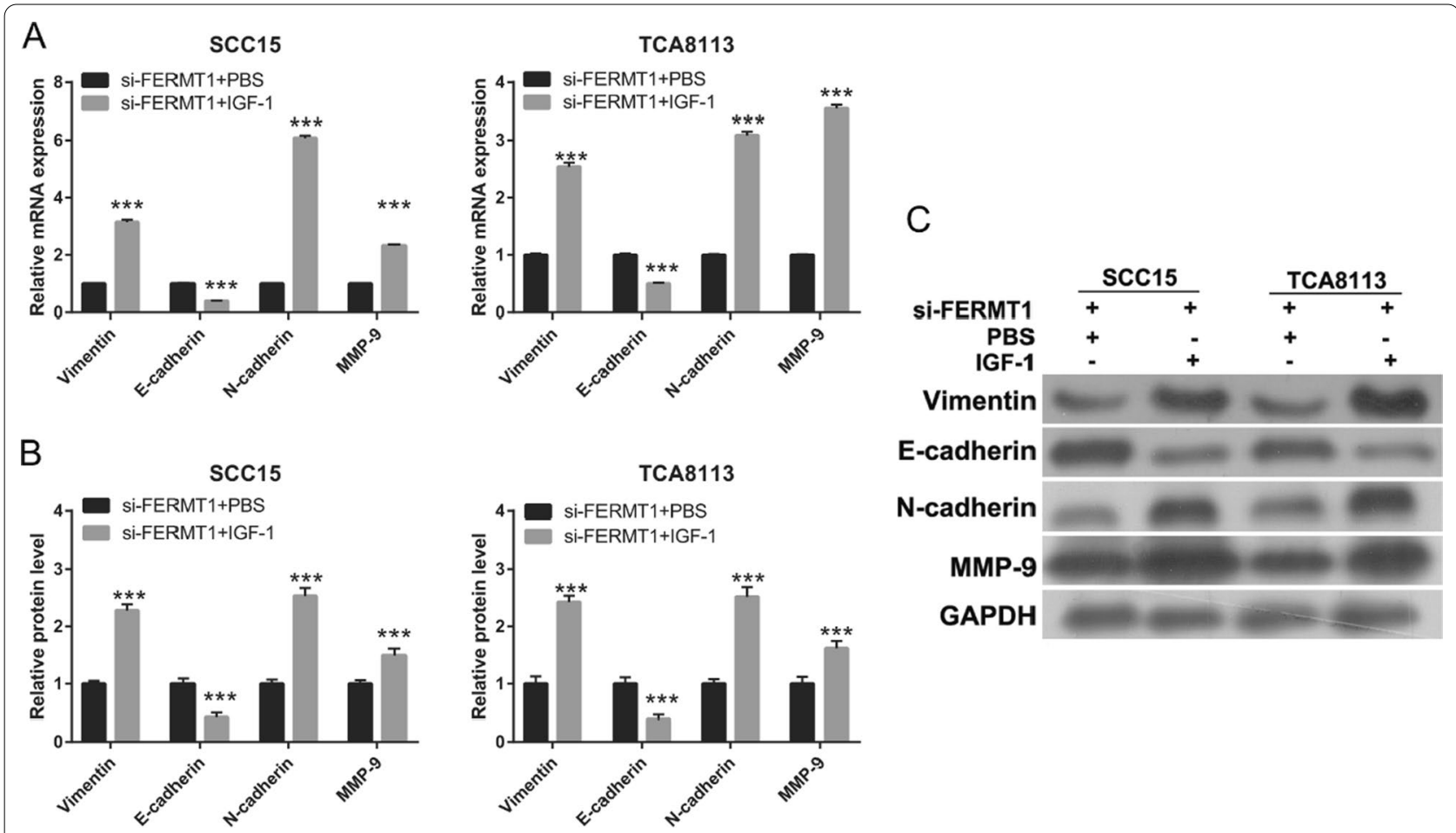

Fig. 7 EMT was promoted after IGF-1 treatment at $24 \mathrm{~h}$ in si-FERMT1-transfected Tca8113 and SCC15 cells. OSCC cells were treated with $5 \mathrm{ng} /$ $\mathrm{ml}$ recombinant human TGF- $\beta 1$ protein. (A, B) The mRNA expression (A) and protein levels (B) of mesenchymal biomarkers including vimentin, $\mathrm{N}$-cadherin, and MMP-9 and epithelial biomarkers including E-cadherin were measured by qRT-PCR and western blotting. (C) The representative image of western blot. ${ }^{* *} P<0.001$, si-FERMT1 + IGF-1 vs si-FERMT1 + PBS 
E-cadherin expression, suggesting that FERMT1 silencing inhibited EMT in TGF- $\beta 1$-induced OSCC cells.

The PI3K/AKT pathway has been noted in OSCC and regulates cancer invasion, metastasis, and EMT [30]. In this study, we found that FERMT1 silencing inhibited PI3K and p-AKT expression, suggested that knockdown of FERMT1 silenced PI3K/AKT pathway. Additionally, activation of the PI3K/AKT signaling pathway reversed the effect of FERMT1 silencing on OSCC cell migration, invasion, and EMT. These results showed that FERMT1 regulated the migration, invasion, and EMT in TGF- $\beta 1$-induced OSCC cells by activating the PI3K/AKT signaling pathway.

\section{Conclusion}

Silencing of FERMT1 inhibits the migration, invasion, and EMT in TGF- $\beta 1$-induced OSCC cells via inactivation of the PI3K/AKT signaling pathway, suggesting that FERMT1 is a novel and potential therapeutic target for anti-metastatic strategies for OSCC.

\section{Abbreviations \\ FERMT1: Fermitin family member 1; OSCC: Oral squamous cell cancer; EMT: Epithelial-mesenchymal transition; TGF- $\beta 1$ : Transforming growth factor- $\beta 1$; MMP-9: Matrix metalloproteinase 9; IGF-1: Insulin-like growth factor-1.}

\section{Acknowledgements}

None.

\section{Authors' contributions}

XW and QC conceived and designed the present study, and developed the methodology, performed the experiments, collected the data, analyzed and interpreted the data. XW drafted the manuscript and QC revised the manuscript. All authors read and approved the final manuscript.

\section{Funding}

None.

\section{Availability of data and materials}

All data generated or analyzed during this study are included in this published article.

\section{Declarations}

Ethics approval and consent to participate

Not applicable.

\section{Consent for publication}

Not applicable.

\section{Competing interests}

The authors declare that they have no competing interests.

\section{Author details}

${ }^{1}$ Department of Stomatology, The First Affiliated Hospital, Guangxi University of Science and Technology, Liuzhou 545006, China. ${ }^{2}$ Medical College, Medical Experimental Center, Guangxi University of Science and Technology, Building D, 257 Liushi Road, Yufeng District, Liuzhou 545006, China.
Received: 6 July 2021 Accepted: 2 November 2021

Published online: 23 November 2021

\section{References}

1. Ferlay J, Shin HR, Bray F, Forman D, Mathers C, Parkin DM. Estimates of worldwide burden of cancer in 2008: GLOBOCAN 2008. Int J Cancer. 2010;127(12):2893-917.

2. Zhang SK, Zheng R, Chen Q, Zhang S, Sun X, Chen W. Oral cancer incidence and mortality in China, 2011. Chin J Cancer Res. 2015;27(1):44-51.

3. Wang M, Xiao C, Ni P, Yu JJ, Wang XW, Sun H. Correlation of betel quid with oral cancer from 1998 to 2017: a study based on bibliometric analysis. Chin Med J. 2018;131(16):1975-82.

4. Chen F, Wang J, Chen J, Yan L, Hu Z, Wu J, Bao X, Lin L, Wang R, Cai L, et al. Serum copper and zinc levels and the risk of oral cancer: a new insight based on large-scale case-control study. Oral Dis. 2018;25(1):80-6.

5. Hu YJ, Chen J, Zhong WS, Ling TY, Jian XC, Lu RH, Tang ZG, Tao L. Trend analysis of betel nut-associated oral cancer and health burden in China. Chin J Dent Res Off J Sci Sect Chin Stomatol Assoc (CSA). 2017;20(2):69-78.

6. Chen F, He BC, Yan LJ, Liu FP, Huang JF, Hu ZJ, Lin Z, Zheng XY, Lin LS, Zhang ZF, et al. Tea consumption and its interactions with tobacco smoking and alcohol drinking on oral cancer in southeast China. Eur J Clin Nutr. 2017;71(4):481-5.

7. D'Souza S, Addepalli V. Preventive measures in oral cancer: An overview. Biomed Pharmacother. 2018;107:72-80.

8. Gupta S, Kong W, Peng Y, Miao Q, Mackillop WJ. Temporal trends in the incidence and survival of cancers of the upper aerodigestive tract in Ontario and the United States. Int J Cancer. 2009;125(9):2159-65.

9. Wikner J, Grobe A, Pantel K, Riethdorf S. Squamous cell carcinoma of the oral cavity and circulating tumour cells. World J Clin Oncol. 2014;5(2):114-24.

10. Yanjia $H$, Xinchun J. The role of epithelial-mesenchymal transition in oral squamous cell carcinoma and oral submucous fibrosis. Clin Chim Acta Int J Clin Chem. 2007;383(1-2):51-6.

11. Zhang J, Zheng G, Zhou L, Li P, Yun M, Shi Q, Wang T, Wu X. Notch signalling induces epithelialmesenchymal transition to promote metastasis in oral squamous cell carcinoma. Int J Mol Med. 2018;42(4):2276-84.

12. Campbell K. Contribution of epithelial-mesenchymal transitions to organogenesis and cancer metastasis. Curr Opin Cell Biol. 2018;55:30-5.

13. Jiang W, Zhang C, Zhang X, Sun L, Li J, Zuo J. CircRNA HIPK3 promotes the progression of oral squamous cell carcinoma through upregulation of the NUPR1/PI3K/AKT pathway by sponging miR-637. Ann Transl Med. 2021;9(10):860.

14. Li C, Lin XF, Wang JN, Ren XS. FBXW7 inhibited cell proliferation and invasion regulated by miR-27a through PI3K/AKT signaling pathway and epithelial-to-mesenchymal transition in oral squamous cell carcinoma. Eur Rev Med Pharmacol Sci. 2020;24(7):3701-9.

15. LV J, Liu C, Chen FK, Feng ZP, Jia L, Liu PJ, Yang ZX, Hou F, Deng ZY. M2-like tumour-associated macrophage-secreted IGF promotes thyroid cancer stemness and metastasis by activating the PI3K/AKT/mTOR pathway. Mol Med Rep. 2021;24(2)

16. Ma J, Sawai H, Matsuo Y, Ochi N, Yasuda A, Takahashi H, Wakasugi T, Funahashi H, Sato M, Takeyama H. IGF-1 mediates PTEN suppression and enhances cell invasion and proliferation via activation of the IGF-1/ PI3K/Akt signaling pathway in pancreatic cancer cells. J Surg Res. 2010;160(1):90-101.

17. Vander Broek R, Mohan S, Eytan DF, Chen Z, Van Waes C. The PI3K/Akt/ mTOR axis in head and neck cancer: functions, aberrations, cross-talk, and therapies. Oral Dis. 2015;21(7):815-25.

18. Liu CC, Cai DL, Sun F, Wu ZH, Yue B, Zhao SL, Wu XS, Zhang M, Zhu XW, Peng $Z \mathrm{H}$, et al. FERMT1 mediates epithelial-mesenchymal transition to promote colon cancer metastasis via modulation of beta-catenin transcriptional activity. Oncogene. 2017;36(13):1779-92.

19. Chen IC, Chiang WF, Huang HH, Chen PF, Shen YY, Chiang HC. Role of SIRT1 in regulation of epithelial-to-mesenchymal transition in oral squamous cell carcinoma metastasis. Mol Cancer. 2014;13(254):1476-4598.

20. Quan J, Elhousiny M, Johnson NW, Gao J. Transforming growth factorbeta1 treatment of oral cancer induces epithelial-mesenchymal transition 
and promotes bone invasion via enhanced activity of osteoclasts. Clin Exp Metastasis. 2013;30(5):659-70.

21. Ouyang Y, Jiang F, Zeng B, Wei C, Yu D. miR-222 knockdown suppresses epithelial-to-mesenchymal transitionin human oral squamous cell carcinoma. Int J Clin Exp Pathol. 2017;10(11):11251-9.

22. Fan J, Yan D, Teng M, Tang H, Zhou C, Wang X, Li D, Qiu G, Peng Z. Digital transcript profile analysis with aRNA-LongSAGE validates FERMT1 as a potential novel prognostic marker for colon cancer. Clin Cancer Res Off J Am Assoc Cancer Res. 2011:17(9):2908-18.

23. Kiriyama K, Hirohashi Y, Torigoe T, Kubo T, Tamura Y, Kanaseki T, Takahashi A, Nakazawa E, Saka E, Ragnarsson C, et al. Expression and function of FERMT genes in colon carcinoma cells. Anticancer Res. 2013;33(1):167-73.

24. Sin $S$, Bonin F, Petit V, Meseure D, Lallemand F, Bièche I, Bellahcène $A$, Castronovo V, de Wever O, Gespach C, et al. Role of the focal adhesion protein kindlin-1 in breast cancer growth and lung metastasis. J Natl Cancer Inst. 2011;103(17):1323-37.

25. Mahawithitwong P, Ohuchida K, Ikenaga N, Fujita H, Zhao M, Kozono S, Shindo K, Ohtsuka T, Aishima S, Mizumoto K, et al. Kindlin-1 expression is involved in migration and invasion of pancreatic cancer. Int J Oncol. 2013:42(4):1360-6.

26. Scanlon CS, Van Tubergen EA, Inglehart RC, D'Silva NJ. Biomarkers of epithelial-mesenchymal transition in squamous cell carcinoma. J Dent Res. 2013;92(2):114-21.
27. Liu CC, Cai DL, Sun F, Wu ZH, Yue B, Zhao SL, Wu XS, Zhang M, Zhu XW, Peng ZH, et al. FERMT1 mediates epithelial-mesenchymal transition to promote colon cancer metastasis via modulation of $\beta$-catenin transcriptional activity. Oncogene. 2017;36(13):1779-92.

28. Kong J, Du J, Wang Y, Yang M, Gao J, Wei X, Fang W, Zhan J, Zhang H. Focal adhesion molecule Kindlin-1 mediates activation of TGF- $\beta$ signaling by interacting with TGF- $\beta R$ I, SARA and Smad3 in colorectal cancer cells. Oncotarget. 2016;7(46):76224-37.

29. Fan H, Zhang S, Zhang Y, Liang W, Cao B. FERMT1 promotes gastric cancer progression by activating the NF-KB pathway and predicts poor prognosis. Cancer Biol Ther. 2020;21:1-11.

30. Harsha C, Banik K, Ang HL, Girisa S, Vikkurthi R, Parama D, Rana V, Shabnam B, Khatoon E, Kumar AP, et al. Targeting AKT/mTOR in oral cancer: mechanisms and advances in clinical trials. Int J Mol Sci. 2020;21(9):3285.

\section{Publisher's Note}

Springer Nature remains neutral with regard to jurisdictional claims in published maps and institutional affiliations.
Ready to submit your research? Choose BMC and benefit from:

- fast, convenient online submission

- thorough peer review by experienced researchers in your field

- rapid publication on acceptance

- support for research data, including large and complex data types

- gold Open Access which fosters wider collaboration and increased citations

- maximum visibility for your research: over 100M website views per year

At BMC, research is always in progress.

Learn more biomedcentral.com/submissions 